\title{
A novel strategy to derive iPS cells from porcine fibroblasts
}

\author{
RUAN WeiMin ${ }^{1}$, HAN JianYong ${ }^{1,2}$, LI Pin $^{2}$, CAO SuYing ${ }^{1,2}$, AN Yang ${ }^{1}$, \\ LIM Bing $^{2} \&$ LI Ning ${ }^{*}$ \\ ${ }^{1}$ State Key Laboratory for Agrobiotechnology, College of Biological Sciences, China Agricultural University, Beijing 100193, China; \\ ${ }^{2}$ Stem Cell and Developmental Biology, Genome Institute of Singapore, 138672, Singapore
}

Received March 12, 2011; accepted April 23, 2011

\begin{abstract}
Induced pluripotent stem (iPS) cell technology demonstrates that somatic cells can be reprogrammed to a pluripotent state by over-expressing four reprogramming factors. This technology has created an interest in deriving iPS cells from domesticated animals such as pigs, sheep and cattle. Moloney murine leukemia retrovirus vectors have been widely used to generate and study mouse iPS cells. However, this retrovirus system infects only mouse and rat cells, which limits its use in establishing iPS cells from other mammals. In our study, we demonstrate a novel retrovirus strategy to efficiently generate porcine iPS cells from embryonic fibroblasts. We transfected four human reprogramming factors (Oct4, Sox2, Klf4 and Myc) into fibroblasts in one step by using a VSV-G envelope-coated pantropic retrovirus that was easily packaged by GP2-293 cells. We established six embryonic stem (ES)-like cell lines in human ES cell medium supplemented with bFGF. Colonies showed a similar morphology to human ES cells with a high nuclei-cytoplasm ratio and phase-bright flat colonies. Porcine iPS cells could form embryoid bodies in vitro and differentiate into the three germ layers in vivo by forming teratomas in immunodeficient mice.
\end{abstract}

induced pluripotent stem cells, Moloney murine leukemia retrovirus vectors, embryoid body, teratoma

Citation: Ruan W M, Han J Y, Li P, et al. A novel strategy to derive iPS cells from porcine fibroblasts. Sci China Life Sci, 2011, 54: 553-559, doi: $10.1007 / \mathrm{s} 11427-011-4179-5$

Porcine induced pluripotent stem (iPS) cells have great potential in genetically engineered porcine production, human disease modeling and tissue transplantation [1]. However, porcine embryonic stem (ES) cells that originate from the inner cell mass (ICM) fail to be established because of a lack of knowledge on pre-implantation development and culture conditions in vitro. Recently, numerous papers report that iPS cells can be directly established by ectopic expression of four reprogramming factors (Oct4, Sox2, Klf4 and $\mathrm{Myc}$ ) in somatic cells from mice [2], humans [3], rats [4], monkeys [5] and Tibetan miniature pigs [6]. Also, iPS cells have been used for nuclear transfer [7], precise gene targeting [8] and disease modeling [9].

Among the reprogramming gene deliver strategies [10],

*Corresponding author (email: ninglcau@ cau.edu.cn) the Moloney murine leukemia derived retrovirus (MMLV) vector pMXs is widely used to efficiently generate and study iPS cells $[2,3,11,12]$. This technique can efficiently introduce and integrate stable transgenes into the genome of any mitotic mammal cell type. The retrovirus can fall into several categories according to the viral envelop and recognized cell type. Ecotropic virus recognizes a receptor found only on mouse and rat cells. Amphotropic virus recognizes a receptor on a broad range of mammalian cell types. Pantropic virus coated by VSV-G protein infects mammal and non-mammal cells. VSV-G protein mediates viral entry via lipid binding and plasma membrane fusion. MMLV retrovirus vectors in mouse and human iPS cells are transcriptionally silent, which suggests complete induced pluripotent reprogramming $[3,11,12]$. In addition, monitoring exogenous gene expression can be easily performed by real-time 
quantitative-PCR (RT-qPCR).

However, ecotropic pMXs retrovirus produced in PLAT-E packaging cells for mouse iPS cell generation yields low transfection efficiencies in non-mouse cells [3], although this limitation may be overcome by introducing a Slc7a1 receptor. In the present study, we describe a novel method to easily deliver pMXs retroviral vectors in one step to efficiently generate iPS cells from porcine somatic cells.

\section{Materials and methods}

\subsection{Fibroblast derivation and culture}

Porcine embryonic fibroblasts were derived from day 26-30 embryos using a standard procedure, maintained in highglucose Dulbecco's Modified Eagle Medium (DMEM; Cat\# 11960, Gibco, Invitrogen, USA) supplemented with $10 \%$ Fetal Bovine Serum (FBS; Cat\# 10099, Gibco, Invitrogen, USA) and $1 \%$ nonessential amino acids (NEAA, Cat\# 11140-050, Invitrogen, USA) and incubated at $37^{\circ} \mathrm{C}$ with $5 \% \mathrm{CO}_{2} . \mathrm{SW} 3$ porcine fibroblasts from black male embryo were used to derive iPS cells. One day prior to retrovirus infection, passage $10 \mathrm{SW} 3$ fibroblasts were plated at $0.1 \times 10^{6}$ cells per well in six-well-plates. MEFs were derived from CF-1 mouse 13.5 days post coitum (d.p.c) embryos and cultured in DMEM supplemented with $10 \%$ FBS, $1 \mathrm{mmol} \mathrm{L}^{-1}$ L-glutamine (Cat\# 2503-081, Invitrogen, USA) 1\% NEAA, $0.1 \mathrm{mmol} \mathrm{L}^{-1} \beta$-mercaptoethanol (Cat\# 21985023, Invitrogen, USA) and 1\% penicillin/streptomycin (Cat\# 15140122, Invitrogen, USA). Passage 4 MEFs (mouse embryo fibroblasts) were mitotically inactivated by $10 \mu \mathrm{g} \mathrm{mL} \mathrm{m}^{-1}$ mitomycin C (Sigma, USA) for 2.5-3 $\mathrm{h}$ and then frozen in $10 \%$ dimethyl sulfoxide (DMSO; Cat\# 2650, Sigma, USA) at $6 \times 10^{6}$ cells per vial. For iPS and ES cell culture, a vial of inactivated MEFs was thawed and plated into four six-well plates or $10 \mathrm{~cm}$ dishes. All reagents for cell culture were purchased from Invitrogen, USA.

\subsection{Retrovirus production and porcine iPS generation}

Retrovirus vectors were purchased from Addgene (www. addgene.com), pMXs-hOct3/4 (Cat\# 17217), pMXs-hSox2 (Cat\# 17218), pMXs-hKlf4 (Cat\# 17219) and pMXs-hcMYC (Cat\# 17220). VSV-G coated retrovirus was produced in GP2-293 cells according to the manufacturer's instructions (Clontech, Germany). Briefly, $12 \mu \mathrm{g}$ pMXs-vector and $6 \mu \mathrm{g}$ pVSV-G were co-transfected into GP2-293 cells by Lipofectamine 2000 (Cat\# 11668019, Invitrogen, USA) at a ratio of $1 \mu \mathrm{g}: 2.5 \mu \mathrm{L}$, which were passaged $<24 \mathrm{~h}$ before transfection without antibiotics. After 8-12 h, the medium was replaced with fresh fibroblast medium. After 48-72 h, supernatant containing virus was collected, filtered through a $0.45 \mu \mathrm{m}$ pore-size membrane and concentrated by ultracentrifugation at $100000 \times g$ (Beckman). Retrovirus was used to infect fibroblasts for 6-12 $\mathrm{h}$ in the presence of $4-8 \mu \mathrm{g}$ $\mathrm{mL}^{-1}$ polybrene (Sigma). After recovery for $1-2 \mathrm{~d}$, infected porcine fibroblasts were trypsinized and seeded at $4 \times 10^{5}$ cells per $10 \mathrm{~cm}$ inactivated MEF-coated plate followed by changing to an ES cell culture condition on the second day. iPS cell derivation medium was based on human serumfree and mouse ES cell culture media. ES cell-like colonies were passaged at day 15-20 post-infection in human ES cell medium and expanded in mouse ES:human ES cell $(1: 1, \mathrm{v} / \mathrm{v})$ medium. Mouse ES cell medium was DMEM supplemented with 15\% FBS (Cat\# 16141079, Invitrogen, USA), 1 mmol L ${ }^{-1}$ L-glutamine, $1 \%$ NEAA, $0.1 \mathrm{mmol} \mathrm{L}^{-1}$ $\beta$-mercaptoethanol and $1000 \mathrm{U} \mathrm{mL}^{-1}$ Leukemia inhibitory factor (LIF, Cat\# ESG1107, Millipore, Germany). Human ES cell medium was DMEM/F-12 (DMEM: nutrient mixture F-12= 1:1; Cat\# 11330057, Invitrogen, USA) supplemented with $20 \%$ KnockOut Serum Replacement (KSR; Cat\# 10828028, Invitrogen, USA), $0.5 \mathrm{mmol} \mathrm{L}^{-1} \mathrm{~L}$-glutamine, $1 \%$ NEAA, $0.1 \mathrm{mmol} \mathrm{L}^{-1} \beta$-mercaptoethanol, and $4 \mathrm{ng} \mathrm{\textrm {mL } ^ { - 1 }}$ basic Fibroblast Growth Factor (bFGF; Cat\# 13256029, Invitrogen, USA). Retrovirus production and infection were performed in a Biosafety Level 2 (BL2) facility.

\subsection{RNA extraction and R-qPCR}

Porcine iPS cells were trypsinized and pelleted followed by washing with cold phosphate buffer solution (PBS, Cat\# 14190, Invitrogen, USA) and resuspended in Trizol (Invitrogen, USA). RNA was extracted using an RNeasy Mini Kit (Cat\# 74106, Qiagen) according to the manufacturer's instructions and reverse-transcribed using a High Capacity cDNA Kit (Cat\# 4368814, Applied Biosystems, USA). Genomic DNA was digested by DNase I (Qiagen). RNA concentration was determined by Nanodrop2000 (Thermo Fisher, USA). RT q-PCR was performed using a SYBR green super mix (Applied Biosystems) and 7900 Q-PCR machine. Primers for pMXs-vectors were as follows $\left(5^{\prime}-3^{\prime}\right)$ : pMXs-1811s (GACGGCATCGCAGCTTGGATACAC) as the common forward primer, Exo-hOct4 (CCCCAGGGCCCCATTTTGGTACC), Exo-hSox2 (GGCACCCCTGGCATGGCTCTTGGCTC), Exo-hKlf4 (ACGATCGTGGCCCCGGAAAAGGACC) and Exo-hMyc (CAACAACCGAAAATGCACCAGCCCCAG). Primers for endogenous factors were Endo-pSox2 (AGGGAAATGGGAGGAGAG and GAGTGTGGATGGGATTGGTG) and Endo-pNanog (CAGTCAAGAAGCAGAAGA and GCTAAGGTTCAGGATGTT). Housekeeping gene GAPDH primers were ACTCACTCTTCTACCTTTGATGCT and TGTTGCTGTAGCCAAATTCA.

\subsection{Immunostaining}

iPS cells were fixed in $4 \%$ paraformaldehyde for $15 \mathrm{~min}$ and permeabilized with $0.1 \%$ Triton X-100 in PBS for 10 min. Cells were then blocked with $6 \%$ bovine serum albu- 
min (BSA, Sigma) in PBS for 30 min followed by washing in $1 \%$ BSA in PBS for 5 min three times. Cells were incubated in primary antibodies $(1: 200)$ in blocking solution overnight at $4^{\circ} \mathrm{C}$. Primary antibodies were purchased from Chemicon, Millipore. After three washes, cells were incubated with a secondary antibody $(1: 200)$ diluted in blocking solution for $30 \mathrm{~min}$ at room temperature. DNA was stained with 4',6-diamidino-2-phenylindole (DAPI) at $300 \mathrm{nmol} \mathrm{L}^{-1}$ for 2-3 min.

\subsection{Karyotype analysis}

Porcine fibroblasts and iPS cells were growth arrested in $100 \mathrm{ng} \mathrm{mL}^{-1}$ colcemid (Karyomax, Cat\# 10092, Invitrogen, USA) for $4 \mathrm{~h}$. Cells were collected and treated with 0.075 mol $\mathrm{L}^{-1} \mathrm{KCl}$ for $30 \mathrm{~min}$. After centrifugation at $1000 \mathrm{r}$ $\min ^{-1}$, for $10 \mathrm{~min}$, cell pellets were fixed in $1: 3$ (glacial acetic acid:methanol) for $10 \mathrm{~min}$ three times. For metaphase analysis, fixed cells were dropped on cold slides, dried at $70^{\circ} \mathrm{C}$ for $3 \mathrm{~h}$, then digested in $0.01 \%$ trypsin at $37^{\circ} \mathrm{C}$ and stained with $10 \%$ Giemsa (Invitrogen, USA).

\subsection{Embryoid bodies formation assay}

Porcine iPS cells were trypsinized into a single cell suspension, seeded on low-adhesive $10 \mathrm{~cm}$ culture plates and cultured in embryoid body forming medium consisting of DMEM/F12, 20\% Knockout Serum Replacement, 1\% pen/strep, $1 \%$ NEAA, $0.5 \%$ L-glutamine and $0.1 \mathrm{mmol} \mathrm{L}^{-1}$ $\beta$-mercaptoethanol. Low-adhesive culture plates were placed on a shaker at $50 \mathrm{r} \mathrm{min}^{-1}$ in a $\mathrm{CO}_{2}$ incubator, which prevented iPS cells from attaching. The culture medium was replaced every day.

\subsection{Teratoma formation}

iPS cells $\left(1 \times 10^{6}\right)$ were subcutaneously injected into the dorsal flanks of SCID (severe combined immunodeficient) mice. After 6-8 weeks, teratomas were dissected and fixed in $10 \%$ formalin phosphate. Samples were embedded in paraffin and sections were hematoxylin \& eosin stained.

\section{Results}

\subsection{Retroviral reprogramming of porcine fibroblasts into ES-like colonies}

Porcine fibroblasts (SW3) were initially infected by ecotropic retrovirus packaged in Plat-E cells. pMXs-GFP as a positive control showed a low infection efficiency of $10 \%-$ $20 \% \mathrm{GFP}^{+}$cells (Figure 1A). This result indicated that porcine fibroblasts do not express the ecotropic virus receptor, which is similar to human cells reported by Yamanaka et al.
[2,3]. We then attempted to generate VSV-G coated pantropic retrovirus produced in GP2-293 cells, which resulted in a higher infection efficiency of $80 \%-90 \% \mathrm{GFP}^{+}$cells (Figure 1A). The GP2-293 cell line is derived from HEK 293 cells and stably expresses viral gap and pol genes, which produces infectious virus by co-transfection of pMXs-vector with pVSV-G [13].

A 100-fold concentrated retrovirus supernatant containing four human factors Oct4/Sox2/Klf4/Myc was used to infect passage 3-6 porcine embryonic fibroblasts (SW3). After 1-2 d recovery, SW3 cells were transferred onto mitomycin $\mathrm{C}$-inactivated mouse embryonic fibroblast (MEF) feeder cells followed by evaluation of various ES cell medium types. ES-like colonies formed and were passaged at day 15-20 (Figure 1B).The derivation scheme is shown in Figure 1A.

During and following reprogramming, we evaluated mouse and human ES cell culture conditions. On day 5-6 after transfection, fibroblasts underwent significant morphology transformation in both types of media, similar to that of human and mouse iPS cell inductions [2,3]. Compact flat ES cell-like colonies appeared by day 10-14, which was consistent with a previous report by Esteban et al. [6]. By day 18-20, only human ES cell culture condition could maintain the morphology of regular human ES-like colonies, whereas differentiated and necrotic cells populated the mouse ES cell culture condition. This result indicated that porcine iPS cell characteristics may resemble human ES cells. Colonies cultured under the human ES cell condition were mechanically passaged onto MEF-seeded 12-well plates. Surviving colonies retained the original cell morphology and could be expanded further. Six healthy iPS cell lines were established. Approximately 200 colonies showed alkaline phosphatase positive per 40000 parental fibroblasts in a side-by-side experiment (data not shown), indicating a $0.5 \%$ reprogramming efficiency.

\subsection{Porcine iPS cells share similar characteristics with human ES cells}

iPS cell lines maintained in human ES cell medium $(20 \%$ knockout serum-replacement supplemented with $4 \mathrm{ng} \mathrm{mL}{ }^{-1}$ bFGF) rapidly proliferated, and exhibited a flat morphology, clear boundaries and a high nuclei to cytoplasm ratio, which are typical human ES cell characteristics. Porcine iPS cell lines were passaged every three days at a 10-15 split ratio using a method for human ES cell passage [14]. The method involves collagenase IV (Invitrogen, USA) treatment for 10-15 min followed by mechanical passaging. All cell lines were subcultured beyond 20 passages and maintained a normal 38 XY karyotype compared with that of the original fibroblasts (Figure 2A). Long-term chromosomal stability in culture is essential for iPS cell use in producing transgenic animals. 

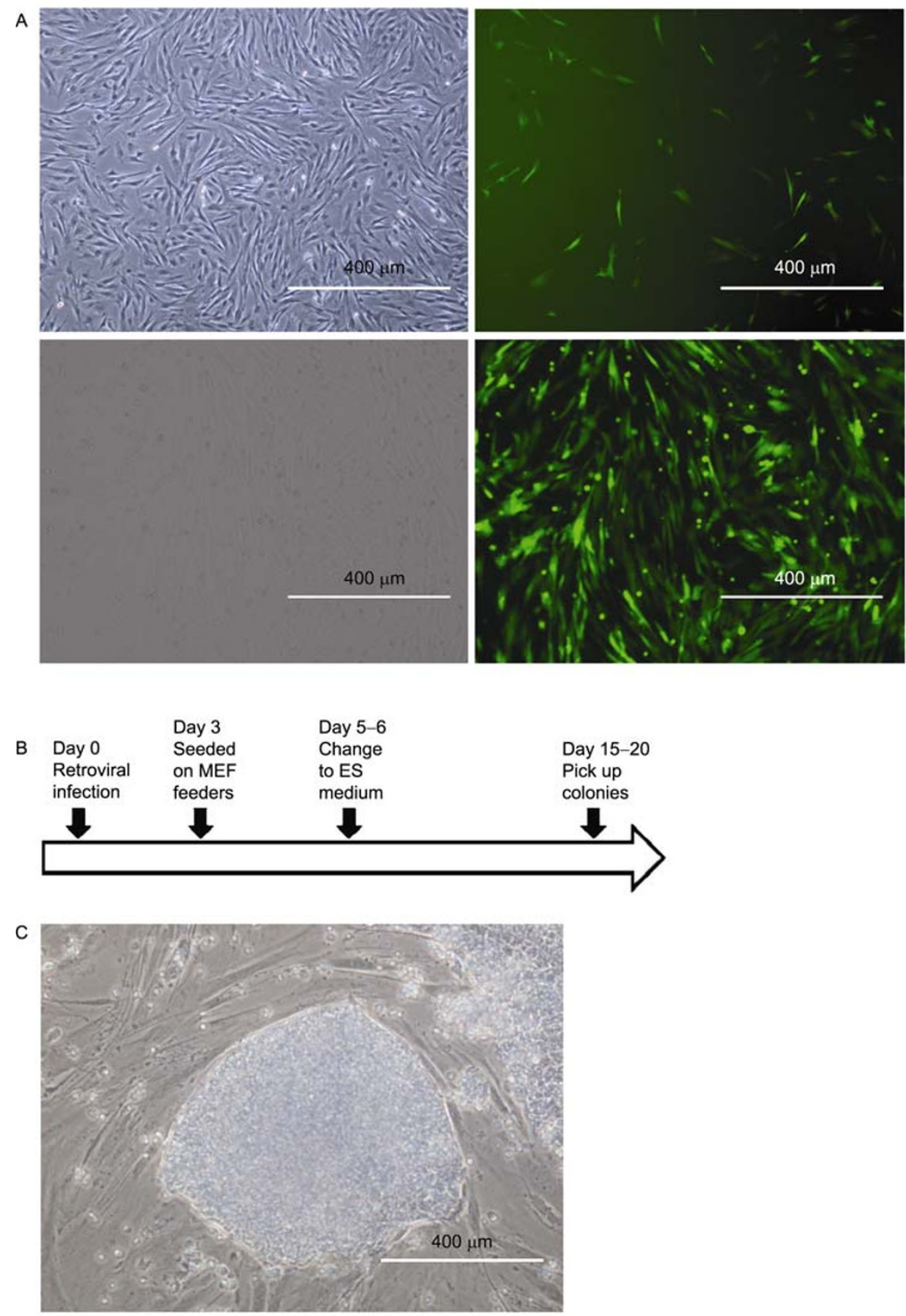

Figure 1 Porcine iPS cell generation using a pantropic retroviral system. A, SW3 porcine fibroblasts infected by ecotropic retrovirus (upper) and pantropic retrovirus (lower), respectively. Images, 100×. B, Timelines for reprogramming by pantropic retrovirus transfection. C, Typical human ES cell-like colonies appeared by day 15 under human ES cell culture condition. Cells were visualized by a NIKON inverted microscope TS100, 100x.

Induced porcine iPS cells expressed numerous common protein and cell surface carbohydrate antigens similar to that of human ES cells. Immunocytochemistry indicated strong ES cell marker expression including Oct4 and Sox2, and stem cell surface markers SSEA-3, SSEA-4 and Tra-1-60. However, the pluripotency marker Nanog was weakly detected by immunostaining, which was consistent with RT-qPCR data (Figure 3B).

\subsection{Activation of endogenous pluripotent genes in por- cine iPS cells}

We analyzed the gene expression of reprogramming factors by RT-qPCR and found significant decreases compared with that of porcine fibroblasts at two days post-infection, which corresponded to the highest human $O / S / K / M$ transgene expression level. These results showed that exogenous genes remained at a high level compared with previous re- 
A

$$
\begin{aligned}
& \frac{11}{1} \frac{\pi 1}{2}-\frac{37}{3} \frac{11}{4}-\frac{\pi}{5} \\
& \frac{11}{6} \frac{11}{7} \frac{11}{8} \frac{11}{9} \frac{:}{10} \\
& \text { 111 } \frac{\text {.8 }}{12} \frac{K}{13} \frac{11}{14} \frac{\text { II }}{15} \\
& \frac{\mu}{16} \frac{\text { แ }}{17} \frac{\text { แ }}{18} \\
& \text { Karyotype: } 38, \mathrm{XY}
\end{aligned}
$$

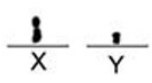

B
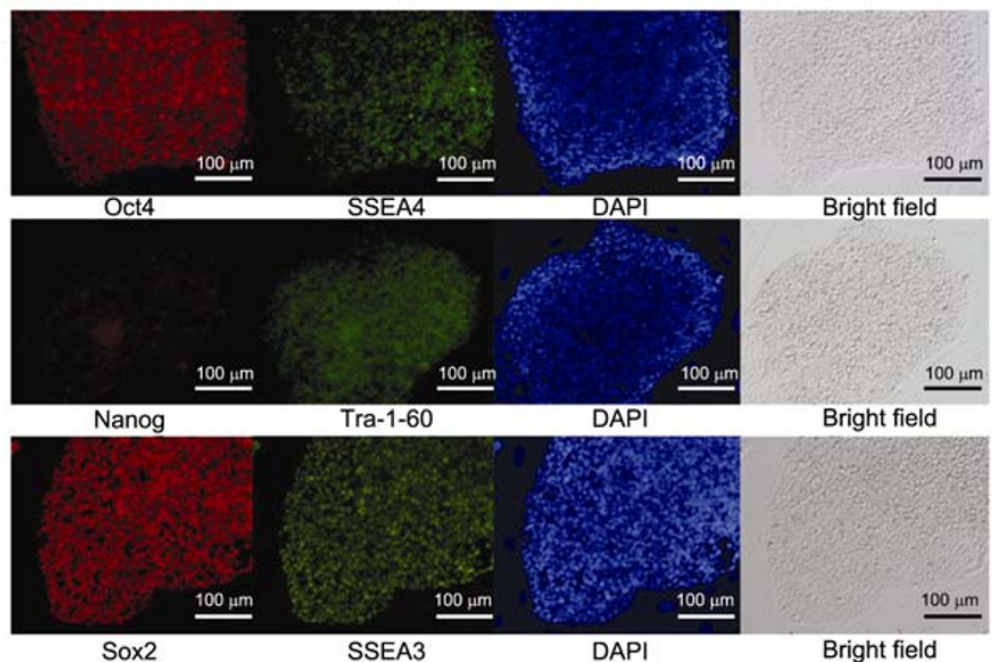

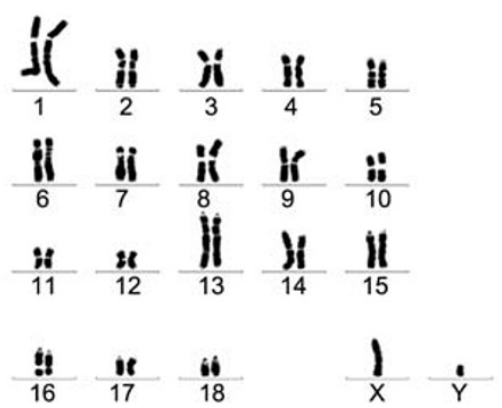

$\frac{16}{16}-\frac{18}{18}$

Figure 2 A, Representative images of passage 4 porcine iPS cell normal karyotypes (right) compared with that of original fibroblasts (left). Image, 600×. B, Immunostaining of porcine iPS cells for stem cell markers. Cells were visualized by a NIKON inverted fluorescence microscope Ti, $200 \times$.
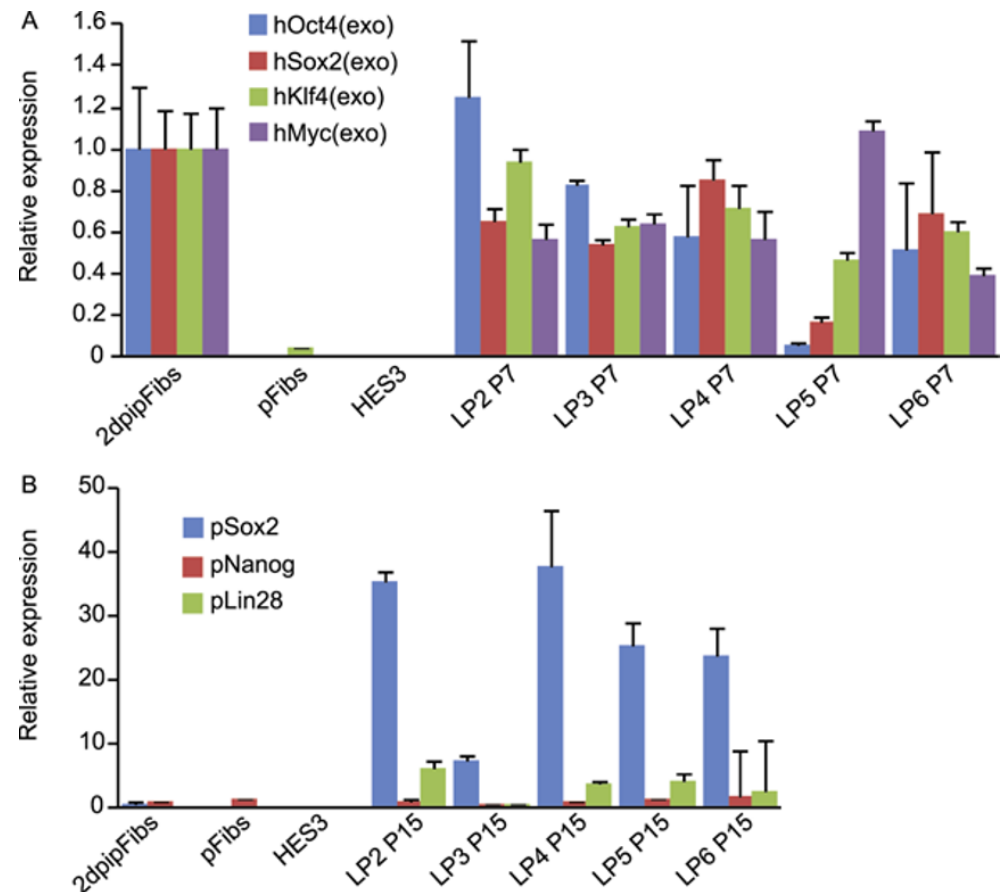

Figure 3 A, Gene expression analysis of porcine iPS cells. RT-qPCR analysis of retrovirus vector expression relative to infected fibroblasts. Day 2 infected porcine fibroblasts (2dpipFibs) were used as a positive control, which expressed the highest level of reprogramming factors. pFibs, pig fibroblasts without virus infection were used as a negative control; HES3 cells show that the PCR primers were vector-specific. LP 2-6 P7 represent passage 7 porcine iPS cell lines. B, RT-qPCR analysis of endogenous pluripotency-related gene expression in passage 15 fibroblasts and iPS cells. HES3 cells show that the PCR primers were porcine endogenous-specific. All data were presented as mean \pm SD. 
ports $[6,15]$. Retrovirus silencing in mouse and human iPS cells is an important event [11], which indicates endogenous gene maintenance of pluripotency in fully reprogrammed iPS cells [12]. Thus, iPS cells generated by this system demonstrated partial reprogramming. Exogenous genes down-regulation can result in abnormal morphology and differentiation as reported by a previous porcine iPS cell study [16], which used doxycyline-induced lentivirus vectors. In our novel pMXs retroviral system, maintained exogenous gene expression in iPS cells conferred "stemness" and could be analyzed by RT-qPCR to elucidate culture conditions that were specific to porcine cells.

\subsection{In vitro and in vivo differentiation of porcine iPS cells}

In vitro and in vivo differentiation potentials are the major characteristic of pluripotent stem cell lines [2]. To test whether porcine iPS cells could form embryoid bodies (EBs), we cultured porcine iPS cell lines in ES cell culture medium without bFGF on low-adhesive culture plates. All six iPS cell lines were able to form EBs in 4-6 d (Figure 4A), indicating a capacity to differentiate in vitro.

To examine whether iPS cells could differentiate into the three germ layers in vivo, we injected the sw3-hOSKM lp4 iPS cell line into the dorsal flanks of SCID mice. After two months, tumors developed and contained various tissue types as determined by histological examination (Figure 4B) including endoderm, mesoderm and ectoderm.

\section{Discussion}

Various methods for exogenous reprogramming factor delivery have been used to generate mouse and human iPS cells, which have been recently reviewed by Maherali et al. [10] and O'Malley et al. [17]. Retrovirus vectors [3], constitutive and dox-inducible lentivirus [18] can yield high gene expression and iPS cell derivation efficiencies. However, these vectors also permanently integrate into the ge-
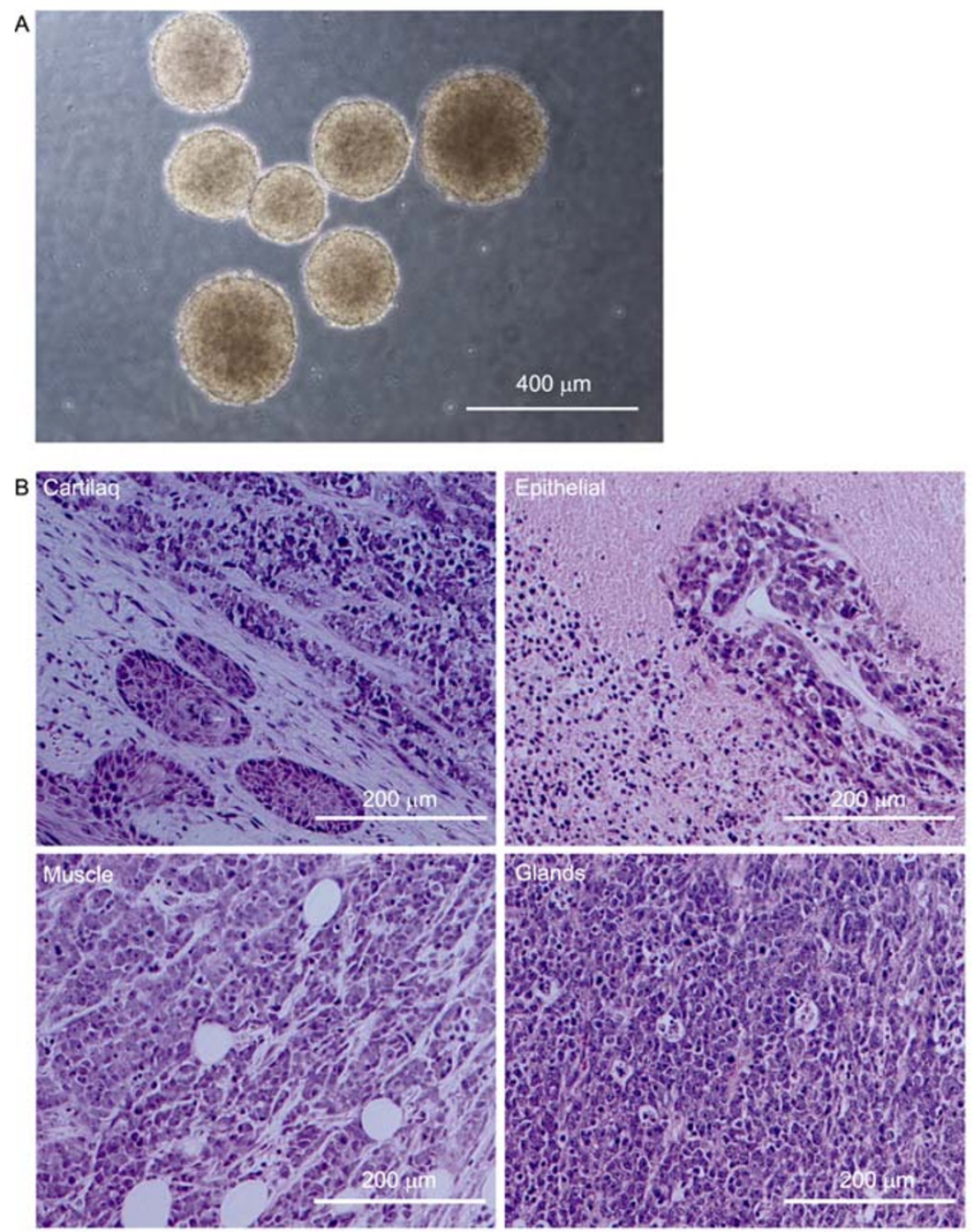

Figure 4 In vitro and in vivo differentiation of porcine iPS cells. A, EB formation after $6 \mathrm{~d}$ suspension culture. B, Histological sections of tumors grown in SCID mince injected with the SW3-hOSKM LP4 cell lines. Differentiated tumors contain tissues derived from the three germ layers including cartilage (mesoderm), gut-like epithelia (ectoderm), muscle (endoderm) and glands (endoderm). Image, 400×. 
nome causing genetic alteration that may affect iPS cell use in clinical applications. Thus, iPS cell production without genetic modification and complete pluripotency is ideal. Excisable piggyBac (PB) [19] and Cre-loxP [20] systems can delete exogenous genes after reprogramming to derive transgene-free iPS cells. Although, a second round of transposase expression in the PB system may alter the genome resulting in chromosomal abnormality. In addition, the validation of factor-excised clones is time-consuming. Adenovirus [21], transient transfection [20] and protein transduction [22] rarely cause genome alteration yielding "clean" iPS cells, which increases safety in the clinic. However, low reprogramming efficiencies limit their use in the derivation and study of iPS cells. Recently, synthetic modified mRNA [23] was shown to have high efficiency and safety in human iPS cell generation.

The MMLV-based retrovirus used in this study was initially used to derive mouse and human iPS cells [2,3]. These retroviral vectors in iPS cells underwent auto-silencing and claimed a pluripotent state. This retrovirus type can be easily produced and yields high infection efficiencies. Moreover, retroviral vector expression can be easily analyzed by RT q-PCR. This strategy allowed us to screen high quality iPS cells with low or no reprogramming factor expression. Also, this method may be applied to other species such as cattle, sheep, dogs, cats and rats.

iPS cell technology has created interest in genetic manipulation such as homologous recombination, gene knockout and knock-in to select and expand positive cell colonies and improve the efficiency of nuclear transfer [7,24]. Porcine ES cells established thus far are not authentic pluripotent stem cells as determined by the mouse ES cell standard of chimera production. There are numerous limitations to overcome before establishing reprogramming factor-independent iPS cells [16]. Other transcription factors may be required for porcine iPS cell induction because authentic ES cells have not been derived from the porcine ICM. Optimal culture conditions that maintain pluripotency and long-term porcine ES cell proliferation remain unknown [25]. Porcine-specific pluripotency and iPS cell selection markers are also unknown. iPS cells derived from somatic cells may delineate these unknown factors.

This work was supported by the National Basic Research Program of China (Grant Nos. 2009CB941003, 2011CBA0110 and 2011CBA01000).

1 Hall V. Porcine embryonic stem cells: A possible source for cell replacement therapy. Stem Cell Rev, 2008, 4: 275-282

2 Takahashi K, Yamanaka S. Induction of pluripotent stem cells from mouse embryonic and adult fibroblast cultures by defined factors. Cell, 2006, 126: 663-676

3 Takahashi K, Tanabe K, Ohnuki M, et al. Induction of pluripotent stem cells from adult human fibroblasts by defined factors. Cell, 2007,
131: 861-872

4 Liao J, Cui C, Chen S, et al. Generation of induced pluripotent stem cell lines from adult rat cells. Cell Stem Cell, 2009, 4: 11-15

5 Liu H, Zhu F, Yong J, et al. Generation of induced pluripotent stem cells from adult rhesus monkey fibroblasts. Cell Stem Cell, 2008, 3: 587-590

6 Esteban M A, Xu J, Yang J, et al. Generation of induced pluripotent stem cell lines from Tibetan miniature pig. J Biol Chem, 2009, 284 : 17634-17640

7 Kou Z, Kang L, Yuan Y, et al. Mice cloned from induced pluripotent stem cells (iPSC). Biol Reprod, 2010, 83: 238-243

8 Hanna J, Wernig M, Markoulaki S, et al. Treatment of sickle cell anemia mouse model with iPS cells generated from autologous skin. Science, 2007, 318: 1920-1923

9 Park I H, Arora N, Huo H, et al. Disease-specific induced pluripotent stem cells. Cell, 2008, 134: 877-886

10 Maherali N, Hochedlinger K. Guidelines and techniques for the generation of induced pluripotent stem cells. Cell Stem Cell, 2008, 3: 595-605

11 Han J, Yuan P, Yang H, et al. Tbx3 improves the germ-line competency of induced pluripotent stem cells. Nature, 2010, 463: 10961100

12 Hotta A, Ellis J. Retroviral vector silencing during iPS cell induction: An epigenetic beacon that signals distinct pluripotent states. J Cell Biochem, 2008, 105: 940-948

13 Yee J K, Friedmann T, Burns J C. Generation of high-titer pseudotyped retroviral vectors with very broad host range. Methods Cell Biol, 1994, 43: 99-112

14 Lerou P H, Yabuuchi A, Huo H, et al. Derivation and maintenance of human embryonic stem cells from poor-quality in vitro fertilization embryos. Nat Protoc, 2008, 3: 923-933

15 Ezashi T, Telugu B P, Alexenko A P, et al. Derivation of induced pluripotent stem cells from pig somatic cells. Proc Natl Acad Sci USA, 2009, 106: 10993-10998

$16 \mathrm{Wu} \mathrm{Z}$, Chen J, Ren J, et al. Generation of pig-induced pluripotent stem cells with a drug-inducible system. J Mol Cell Biol, 2009, 1: 46-54

17 O’Malley J, Woltjen K, Kaji K. New strategies to generate induced pluripotent stem cells. Curr Opin Biotechnol, 2009, 20: 516-521

18 Wernig M, Lengner C J, Hanna J, et al. A drug-inducible transgenic system for direct reprogramming of multiple somatic cell types. Nat Biotechnol, 2008, 26: 916-924

19 Woltjen K, Michael I P, Mohseni P, et al. piggyBac transposition reprograms fibroblasts to induced pluripotent stem cells. Nature, 2009, 458: 766-770

20 Kaji K, Norrby K, Paca A, et al. Virus-free induction of pluripotency and subsequent excision of reprogramming factors. Nature, 2009, 458 $771-775$

21 Zhou W, Freed C R. Adenoviral gene delivery can reprogram human fibroblasts to induced pluripotent stem cells. Stem Cells, 2009, 27: 2667-2674

22 Kim D, Kim C H, Moon J I, et al. Generation of human induced pluripotent stem cells by direct delivery of reprogramming proteins. Cell Stem Cell, 2009, 4: 472-476

23 Warren L, Manos P D, Ahfeldt T, et al. Highly efficient reprogramming to pluripotency and directed differentiation of human cells with synthetic modified mRNA. Cell Stem Cell, 2010, 7: 618-630

24 Zhou S, Ding C, Zhao X, et al. Successful generation of cloned mice using nuclear transfer from induced pluripotent stem cells. Cell Res, 2010, 20: 850-853

25 Chou Y F, Chen H H, Eijpe M, et al. The growth factor environment defines distinct pluripotent ground states in novel blastocyst-derived stem cells. Cell, 2008, 135: 449-461

Open Access This article is distributed under the terms of the Creative Commons Attribution License which permits any use, distribution, and reproduction in any medium, provided the original author(s) and source are credited. 